\title{
Cytomorphological and Cytochemical Identification of Microglia
}

\author{
Subhajit Das Sarma, Koushik Chatterjee, Himadri Dinda, Dhriti Chatterjee, and \\ Jayasri Das Sarma
}

Department of Biological Sciences, Indian Institute of Science Education and Research-Kolkata (IISER-K), Mohanpur Campus, P.O. BCKV Campus Main Office, Mohanpur West Bengal, Nadia, Mohanpur 741252, India

Correspondence should be addressed to Jayasri Das Sarma; dassarmaj@iiserkol.ac.in

Received 7 May 2013; Accepted 18 July 2013

Academic Editors: R. El Ridi and S. M. Varga

Copyright ( 2013 Subhajit Das Sarma et al. This is an open access article distributed under the Creative Commons Attribution License, which permits unrestricted use, distribution, and reproduction in any medium, provided the original work is properly cited.

\begin{abstract}
Microglia is one of the major resident immune cells in the central nervous system and is considered to be the key cellular mediator of neuroinflammatory processes. Identification of different Microglial states of activation by morphologic means has been one of the major challenges in the field of neurobiology of diseases. Therefore, microglial biology demands techniques to identify differing stages of microglia in different neuroanatomic locations as well as understanding the role of Microglia in different Neurological diseases. This present study is aimed towards summarizing the literature and for understanding the progress made in different Cytomorphological and Cytochemical techniques of identifying Microglia. This study also review recently used Immunohistochemistry techniques, along with Ultrastructural studies determining different morphological features of resting to activated phagocytic Microglia in a viral induced experimental animal model of neuroinflammation. Results revealed that chronic Microglial activation is considered to be an important component of neuronal dysfunction, injury, and loss (and hence to disease progression). Thus, Microglial research with special emphasis on identification of different activation states of Microglia has gradually become significant.
\end{abstract}

\section{Introduction}

Microglia, the resident macrophages of the Central Nervous System (CNS), is known to support and sustain proper Neuronal functions. Existence of this Glial cell in CNS has been reported, a century ago [1]. Nissl was the first to recognize Microglia and name it as "stabchenzellen" (rod cells) and considered it as a reactive neuroglia. He also suggested that, Microglia has the capacity of migration and Phagocytosis [1]. Regarding the origin of Microglia, a complete framework was provided for defining this particular cell type by del Rio-Hortega in 1932, but still many of the features remained controversial [2]. Microglia transits through different stages of development to attain its maturity and functionality in the CNS. The first stage is of Ameboid Microglia, and it shares common Immunological, Histochemical and Morphological features with Macrophages outside the CNS [2]. Hence, it is also sometimes termed as the Macrophages of the Brain. The Ameboid microglia is considered to be round in shape or have short and broad processes. It is assumed that cells with Dendrite and elongated morphology also belong to this particular type of Microglia (Ameboid) [3]. In due course of time and under certain circumstances, some parts of the Embryonic Ameboid Microglia gets degenerated and some develop thin processes to become Ramified Microglia in an adult Brain [4]. This transitory phase leads to total disappearance of the preliminary form of Microglia, which is "Ameboid Microglia," thereby providing space for the Ramified Microglia. In response to variety of brain injuries and inflammation, this Ramified Microglia is capable of dramatically changing its structural and chemical morphology into a reactive or an Amedboid Microglia. Along with that, it is also capable of rapidly upregulating a large number of proteins which are receptor types and myriad of secretory products (Figure 1), which acts for defense and potentially causes perturbation to the infected and injured CNS [5].

\section{Mechanism of Activation}

Regarding the mechanism of activation of Microglial cells, it is to convey that very little is known about the metabolic state 

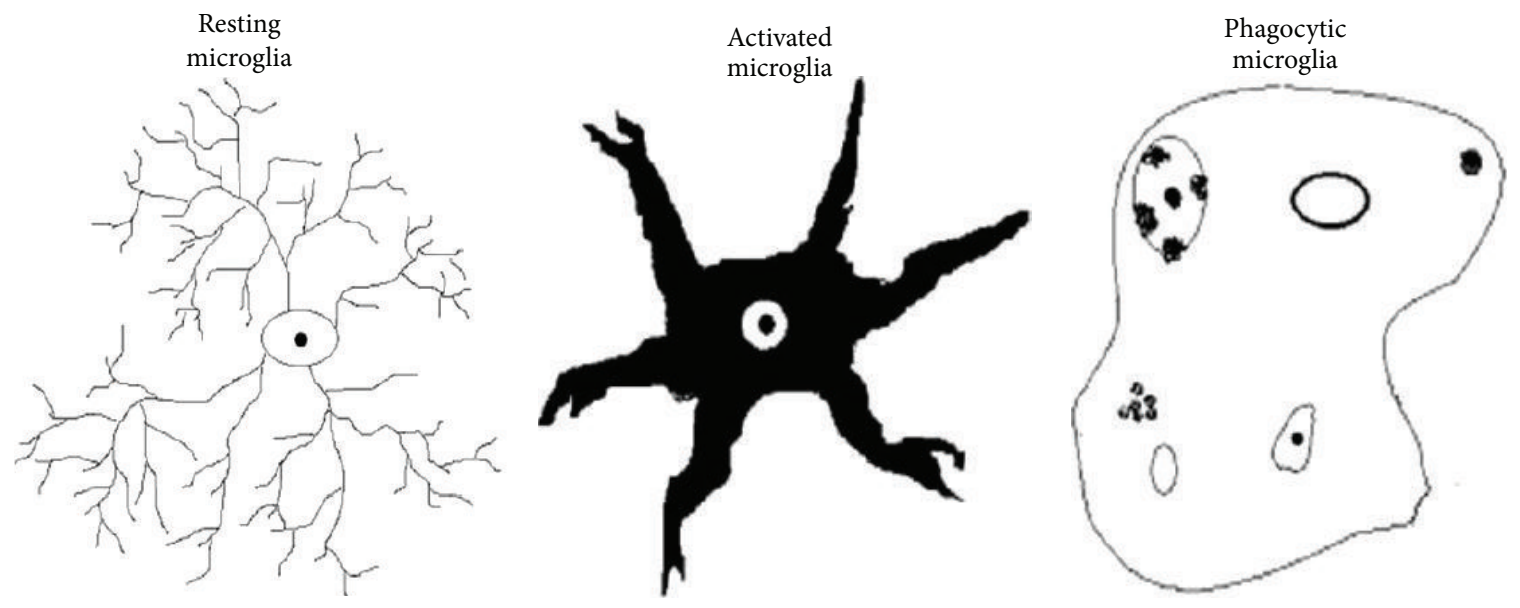

\begin{tabular}{|c|l|c|c|c|}
\hline \multirow{2}{*}{ Sl No } & \multicolumn{1}{|c|}{ Specifications } & Resting & Activated & Phagocytic \\
\cline { 2 - 4 } & \multicolumn{1}{|c|}{} & & \\
\hline 1 & Proliferation & $-/+$ & + & + \\
\hline 2 & Griffonia simplicifolia & & & \\
\hline 3 & B $_{\text {-isolectin }}$ & + & + & + \\
\hline 4 & Vimentin & - & $-/+$ & $+/+$ \\
\hline 5 & Macrophages markers (ED1, ED2, OX-41) & + & + & + \\
\hline 6 & CR3 complement receptor (OX-42) & - & + & + \\
\hline 7 & MHC class I antigen (OX-18) & $-/+$ & + & + \\
\hline 8 & MHC class II (OX-6) & $-/+$ & + & + \\
\hline 9 & CD4 antigen (W3/25) & - & $-/+$ & + \\
\hline 10 & CD8 antigen (OX-8) & - & + & + \\
\hline 11 & Leukocyte common antigen (OX-1) & & & + \\
\hline
\end{tabular}

Figure 1: Different states of Microglia and associated phenotypic characteristics (adopted from Neuroglia; Second Edition; Edited by Helmut Kettenmann and Bruce R. Ransom).

of Resting Microglia. Even then, there are lines of evidence of slow but steady rate of proliferation; it is confirmed from incorporated $\left[{ }^{3} \mathrm{H}\right]$ thymidine in situ in the healthy Brain [6]. The Microglial activation for a long period of time has been evaluated based on the phenomenon of proliferation and expression of immunoregulatory molecules [7]. Some cell surface markers like Major Histocompatibility ComplexII (MHC-II) are important in Immune Regulation and these are constitutively expressed in Ramified Microglia. For understanding the activation mechanism of Microglia, several approaches have been made, both in in vitro and in vivo model of studies. Recent advancement over the past 15 to 20 years in the field of Microglia reveals that its response to different "tissue insults" is in the form of complex array of Inflammatory Cytokines and actions. Furthermore, this action surpasses the previous historical perspective of Phagocytosis and Reactive Gliosis. Nowadays, Microglial research has become the main point of focus in the field of cellular Neuroimmunology and also in the field of neuroinflammation. One of the major challenges in the field of Microglial research is to detect precisely the different states of activation of Microglia by morphologic means. Hence, the field of Microglial Neurobiology demands techniques to identify differing stages of Microglia in different Neuroanatomic locations. The current understanding is based on the expression of surface molecules in Ramified Microglia, Activated and in Phagocytosed Microglia (Figure 1). One contrary of such surface expression is that these molecules are more or less expressed in different stages of Microglia. The only difference lies in their level of expression. In this study, we have reviewed different staining procedure starting from the time of Pio del Rio Hortega (1921). Along with that special emphasis has been provided on the recent Immunohistochemical techniques to identify Microglia in Neuroanatomic location of a viral induced Neuroinflammation in experimental Mouse model [8-11].

2.1. Methods of Identification. Through the understanding of morphological changes, the Microglial role in the diseased CNS is recognized. Hence, identification of Microglia through different staining procedure provided Cytomorphological understanding. Staining of Microglia with silver carbonate started with Pio del Rio Hortega and went through few modifications, where CNS tissues were fixed in formalin and yielded successful staining results [13]. Later there were efforts made with CNS tissues of a wound, where 
the tissue was colored using trypan blue intravitally, which coloured the Macrophages of that particular area. Along with, it also stained other Neuroglia which made it difficult to differentiate the Macrophages from Neuroglial cells and hence required much specialized procedure of staining for differentiation [14]. Formalin-dextran- $\mathrm{CaCl}_{2}$ solution was used as a fixation solution in an attempt of modification of Weil and Davenport's (1933) silver carbonate method for identification of Microglia. This staining method was also able to demonstrate both resting as well as activated Microglia [15]. A rapid and simple impregnation method using ammoniacal silver nitrate in epoxy-embedded tissues demonstrated the morphological aspects of a Reactive Microglia [16].

Identification of the Microglial cells beyond the Cytomorphological understanding was enhanced by the development of different Cytochemical procedures of staining. Cytochemical procedures took advantage of the exclusive expression of certain molecules in specific cell types (Table 1 and Figure 1) [17]. Different Cytochemical methods used acid phosphatase, lysozyme, peroxidase, alpha-naphthyl acetate esterase, alphanaphthyl butyrate esterase, adenosine triphosphatase, and the Periodic Acid-Schiff reaction (PAS reaction). The distinct activity of almost all these enzymes and the PAS reaction were evident in Reactive Microglia [18].

For visualization of cells in vitro and in tissue sections, lectins and antibodies are directly coupled with fluorescent dyes or with avidin/streptavidin peroxidase conjugate for staining microglia [17]. Identification of Microglia with lectins (carbohydrate binding proteins) as a tool is of particular interest as the intensity of staining varies depending on their functional state based on the interaction of various lectins with Microglial membrane [12, 18, 19]. In several studies, it has been shown that Microglia stained intensely with GS-1, RCA, WGA, and ConA and slightly with DBA, UEA, BPA, and SBA, Later most of the lectin based staining was predominantly used for identify Microglial activation state in culture (in vitro) only.

Immunohistochemistry as a technique is based on the expression of a stage-specific protein and the availability of the antibodies against such specific protein as immunogen.

2.2. Identifying Techniques in Use. Immunohistochemistry is the major technique which is being used proficiently to differentiate different stages of CNS Microglia in our Laboratory. In the field of Neuroimmunology and Neurobiology our laboratory is focused on understanding the mechanism of Demyelination in an Experimental Animal model and Immunohistochemistry is one of the most routinely used techniques to identify inflammatory cells in the Virus-induced inflamed CNS tissues as well as isolated CNS cells in culture. This technique is used based on the mostly available antibody against the antigen CD11B which is a known marker for Histochemical analysis of Microglia/Macrophages in mice. CD11B was used in our previous studies to differentiate the Neuroinflammatory status of the CNS in viral infection $[12,19]$. Our further study using CD11B is not consistent enough; hence, Ibal antibody is used [20]. Ibal is a Macrophages/Microglia specific calcium binding protein which is involved in RacGTPase-dependent membrane ruffling and Phagocytosis [20-22]. In our several studies we have noticed that Ibal is a better marker for morphological differentiation of Resting- Ramified Microglia versus Activated-Amoeboid and Phagocytotic Microglia, as it is involved in RacGTPase-dependent membrane ruffling and Phagocytosis [20] (unpublished data from Das Sarma Laboratory).

2.3. Detailed Method of Iba1 Staining. Inflamed Brain tissues were harvested from MHV-A59 or its Isogenic recombinant strain RSA59 both of which can induce acute encephalitis (inflammation in the brain), Meningitis, and Demyelination during chronic stage [8, 19]. MHV-A59 or RSA59 induced Neuropathology mimics certain aspects of Human Demyelinating disease like Multiple Sclerosis (MS). Mock and infected Mice were perfused transcardially with Phosphate Buffer Saline (PBS-7.4 pH) and thereafter fixed with 4\% PFA (PFA-7.2 pH) in 0.1 M sodium phosphate buffer ( $\mathrm{pH} 7.4$ ). Brains were dissected and postfixed in $4 \%$ paraformaldehyde overnight at Room Temperature (RT). All tissues were then processed for paraffin embedding and sectioning in the Histology and Microscopy core at Department of Biology, IISER-Kolkata. Slides were deparaffinized and sections were immersed in Xylene, rehydrated in distilled water, and thereafter treated in high-temperature antigen retrieval or antigen unmasking solution. Briefly, Vector Antigen Unmasking Solutions (Cat. No. H-3301; citrate based) were designed for use in high-temperature unmasking procedures. Antigen Unmasking Solution was shaken well and $9.375 \mathrm{~mL}$ of the concentrated stock Antigen Unmasking Solution is diluted in 1 liter of distilled water into a glass beaker and heated in $90^{\circ} \mathrm{C}$. Slides were positioned into metal staining racks (Note: do not place slides close together; uneven staining may occur) and boiled for 15 minutes into the diluted antigen unmasking solution. Then, slides were removed and immediately placed under tap water. Thereafter, without letting the tissues dry, sections were washed in PBS buffer ( $\mathrm{pH}$ 7.5) for 5 minutes and preceded for Immunohistochemical Labeling. Some tissues may bind avidin, biotinylated horseradish peroxidase, or other Biotin/Avidin System components nonspecifically. This binding may be due to endogenous biotin or biotinbinding proteins, lectins, or nonspecific binding substances present in the section. To avoid such nonspecific binding antigen unmasked tissue sections were incubated for $1 \mathrm{hr}$, with normal Goat Serum. Briefly, one to four drops of stock normal Goat Serum (VECTASTAIN ABC Kit; Catalogue No. 6105, from Vector Laboratories. Inc) in one mL PBS (one drop is approximately $50 \mu \mathrm{L}$ ) is added and then incubated overnight with Rabbit anti-Ibal (1:100; Wako, Richmond, VA, USA). Thereafter tissue sections were incubated with biotinylated secondary antibodies (1:200; Vector Laboratories, Burlingame, CA) at (RT) for 1 hour. Primary and secondary antibody was then diluted in PBS with $1 \%$ normal Serum and the Immunoreactivity was visualized with the Vectastain $A B C$ system and 3,3'-diaminobenzidine (Vector Laboratories, Burlingame, CA, USA). ABC reagent was prepared by adding two drops of $A B C$ Reagent A provided with 
TABLE 1: Surface antigens and thier detection techniques with functional relevance.

\begin{tabular}{|c|c|c|}
\hline Molecular structure/antigen & Detection agent/technique & Properties and functional relevance \\
\hline \multicolumn{3}{|c|}{ Identification* } \\
\hline $\begin{array}{l}\text { Surface carbohydrates (oligosaccharides } \\
\text { containing-galactose residues) }\end{array}$ & $\begin{array}{l}\text { Griffonia (Bandeiraea) simplicifolia } \\
\text { isolectin B4 }\end{array}$ & Not known, also expressed by blood vessels \\
\hline $\begin{array}{l}\text { Surface carbohydrates (oligosaccharides } \\
\text { containing } N \text {-acetylglucosamine } \\
\text { oligomers) }\end{array}$ & $\begin{array}{l}\text { Lycopersicon esculentum (tomato) } \\
\text { lectin }\end{array}$ & Not known, also expressed by blood vessels \\
\hline $\begin{array}{l}\text { Ibal (ionized calcium-binding } \\
\text { adaptormolecule 1) }\end{array}$ & Antibodies against Ibal & Protein with suggested role in calcium homeostasis \\
\hline $\mathrm{CD} 11 \mathrm{~b} / 18(\alpha \mathrm{M} \beta 2$ integrin $)$ & $\begin{array}{l}\text { Antibodies against CD11b/CD18 } \\
\text { (MAC1, OX 42) }\end{array}$ & $\begin{array}{l}\text { Complement receptor } 3 \text { (CR3) with additional binding } \\
\text { properties for other molecules, for example, Fibrinogen }\end{array}$ \\
\hline CD45 (leukocyte common antigen, LCA) & $\begin{array}{l}\text { Antibodies against CD } 45 \text {, low } \\
\text { expression compared with } \\
\text { infiltrating leukocytes }\end{array}$ & $\begin{array}{l}\text { Transmembrane protein tyrosine phosphatase, } \\
\text { functions in signaling, also roles for TCR and BCR } \\
\text { activity }\end{array}$ \\
\hline CD68 (macrosialin) & Antibodies against CD68 & $\begin{array}{l}\text { Role in Phagocytosis and as oxidized LDL-binding } \\
\text { protein }\end{array}$ \\
\hline F4/80 antigen & F4/80 antibody & $\begin{array}{l}\text { Member of the epidermal growth factor } \\
\text { (EGF)-transmembrane } 7 \text { (TM7) family, suggested role } \\
\text { in immune tolerance }\end{array}$ \\
\hline
\end{tabular}

\section{Functional orientation}

MHC II

CD11c (integrin $\alpha \mathrm{X}$ )

CD34 (mucosialin, sialomucin)

iNOS (inducible nitric oxidesynthase)

\begin{tabular}{ll} 
& nitrite in vitro \\
\hline & \multicolumn{1}{c}{ Transgenic models } \\
& Transgenic Mice expressing \\
enhanced green fluorescent protein & (EGFP) in the $C \times 3$ crl locus \\
(fractalkine receptor) & Transgenic mice expressing \\
& enhanced green fluorescent protein \\
& (EGFP) under control of the \\
Ibal & Ibalpromoter
\end{tabular}

Antigen presentation by APC

Cell adhesion, marker for dendritic cells as APC Roles in adhesion, marker for precursor cells of the myeloid lineage

Enzymatic synthesis of NO from arginine during inflammation (upon gene induction), resulting also in citrulline generation

Receptor for the chemokine fractalkine (CX3CL1) with implications for the control of Microglial activity

Protein with suggested role in calcium homeostasis the kit in $10 \mathrm{~mL}$ of PBS, pH 7.5 containing $0.1 \%$ Tween 20 and subsequently adding two drops of $\mathrm{ABC}$ Reagent $\mathrm{B}$ to the $\mathrm{ABC}$ mixing solution immediately. The ABC Reagent was allowed to stand for about 30 minutes before the use of the sections. After the secondary antibody incubation step, sections were rinsed in PBS for three times. ABC reagent made in PBS with $0.1 \%$ Tween 20 was added and incubated for 30 minutes, then after rinsed with PBS for five times. And then the immunoreactivity was visualized with the Vectastain ABC system and 3,3'-diaminobenzidine (Vector Laboratories, Burlingame, CA,USA). Detailed stepwise protocol of Ibal immunostaining is attached in Table 2. All Immunostained sections were photographed, digitally scanned, and analyzed on an Aperio Scan scope XT slide scanner and Aperio Image scope version 10.0.36.1805 software (Aperio). Images were processed using Adobe PhotoshopCS3 (Figures 2, 3 and 4).
2.3.1. Ultrastructural Studies. To characterize the Phagocytiotic Microglia, this study took the advantages of ultrasturctural images available from our studies (Figure 5). Day 30 postinfected inflamed Mice were anesthetized, perfused with 4\% PFA, and spinal cords were harvested and fixed overnight in $2 \%$ glutaraldehyde. Samples for transmission electron microscopy (TEM) were postfixed with $1 \%$ osmium tetroxide, dehydrated, and flat embedded in Poly-Bed 812 epoxy resin (Polysciences). Half-micrometer thick sections were cut from the lesional epicenter, stained with toluidine blue, and examined by light microscopy. Ultrathin TEM sections ( $600 \AA)$ were cut from representative foci of demyelination from the toluidine blue-stained semithin sections and mounted on 200 mesh copper grids, stained with uranyl acetate and bismuth subnitrate, and viewed under a JEOL JEM 1010 (Figure 5). 
TABLE 2: Steps of Ibal immunostaining on paraffin sections by using "vectastain ABC kit (Cat No PK-4001)."

\begin{tabular}{|c|c|c|}
\hline Steps & Time of incubation & Temperature \\
\hline \multicolumn{3}{|l|}{ Deparaffinize slides on a heat block } \\
\hline Deparaffinize with Xylene & 5 mins $* 2$ times & RT \\
\hline $100 \%$ ethyl alcohol & 5 mins & RT \\
\hline $70 \%$ ethyl alcohol & 5 mins & RT \\
\hline Distilled water & 5 mins & $\mathrm{RT}$ \\
\hline PBS 1x & 5 mins & RT \\
\hline $\begin{array}{l}\text { Antigen retrieval: Antigen Unmasking solution (Vector Laboratories; H3300) } \\
(9.375 \mathrm{~mL} \text { of antigen unmasking solution in } 1 \text { liter of distilled water) }\end{array}$ & 15 mins & $95^{\circ} \mathrm{C}$ \\
\hline Blocking (Vector laboratories rabbit kit) $(15 \mu \mathrm{L} / 1 \mathrm{~mL}$ of $\mathrm{PBS})$ & 60 mins & $37^{\circ} \mathrm{C}$ \\
\hline Anti-Ibal antibody (1:100 dilution) & 120 mins & $37^{\circ} \mathrm{C}$ \\
\hline PBS 1x wash & & 3 mins $\times 3$ times \\
\hline Secondary antibody (Vector laboratories rabbit kit) $(5 \mu \mathrm{L} / \mathrm{mL}$ of PBS) & 60 mins & $37^{\circ} \mathrm{C}$ \\
\hline PBS wash & 3 mins & RT \\
\hline $\begin{array}{l}\mathrm{ABC} \text { reagent (Vector laboratories rabbit kit) }(1 \mu \mathrm{L} \text { Reagent } \mathrm{A}+1 \mu \mathrm{L} \text { reagent } \mathrm{B}+ \\
10 \mu \mathrm{L} \text { of } \mathrm{PBS} 1 \mathrm{x})\end{array}$ & 30 mins & $37^{\circ} \mathrm{C}$ \\
\hline $\begin{array}{l}\text { DAB substrate (Vector laboratories rabbit kit) }(500 \mu \mathrm{L} \text { distilled water }+100 \mu \mathrm{L} \\
\left.\text { buffer }+200 \mu \mathrm{L} \text { DAB }+100 \mu \mathrm{L} \mathrm{H}_{2} \mathrm{O}_{2}\right)\end{array}$ & $\begin{array}{l}5-10 \text { mins (till a brown } \\
\text { colour develops) }\end{array}$ & RT \\
\hline Distilled water & 5 mins & \\
\hline Hematoxylin (Counter staining) & $30 \mathrm{sec}$ & \\
\hline \multicolumn{3}{|l|}{ Running water wash } \\
\hline $50 \%$ Ethanol & $10 \mathrm{sec} / 20 \mathrm{dips}$ & \\
\hline $70 \%$ Ethanol & $12 \mathrm{sec} / 24 \mathrm{dips}$ & \\
\hline $100 \%$ Ethanol & $14 \mathrm{sec} / 28 \mathrm{dips}$ & \\
\hline Xylene & 3 mins $\times 3$ times & \\
\hline Mounting with refrax mounting medium and observed under light microscope & & \\
\hline
\end{tabular}

RT: room temperature.

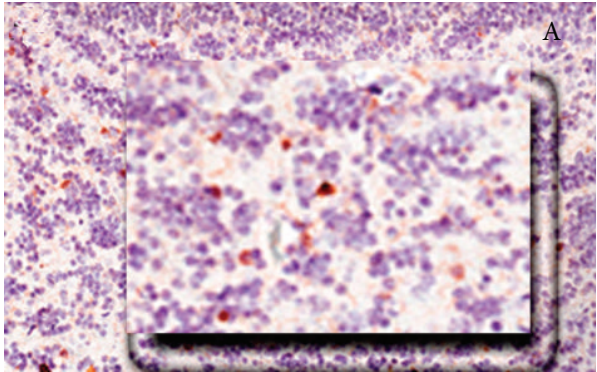

(a)

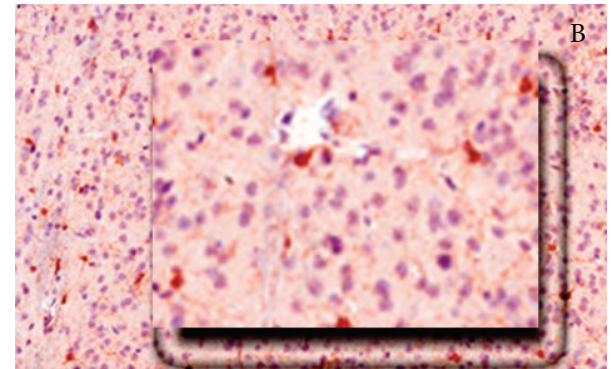

(b)

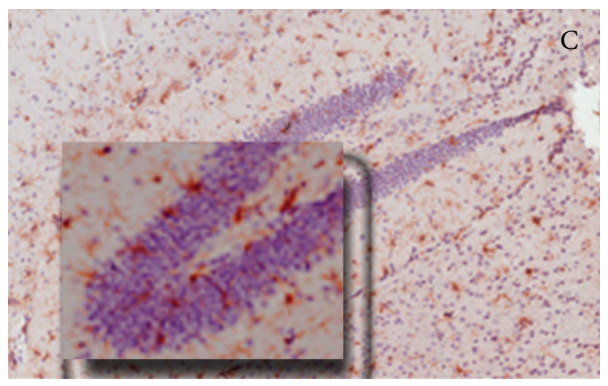

(c)

FIGURE 2: Distribution of ramified microglia in the nonpathological Brain of adult Mouse. 5-micron thick sagittal brain section from 4 weekold C57BL/6 mice were Immunostained with anti-Ibal antibody. Ramified resting Microglia are shown in olfactory bulb (a) (200x), basal forebrain (b) (200x), and hippocampal region (c) (100x). 


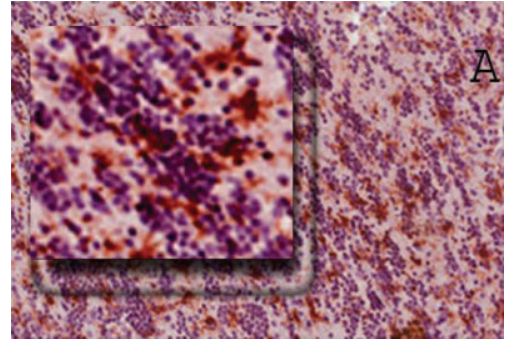

(a)

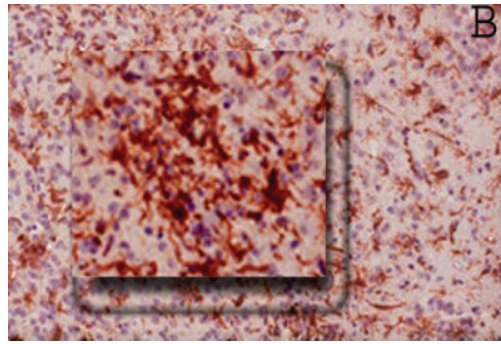

(b)

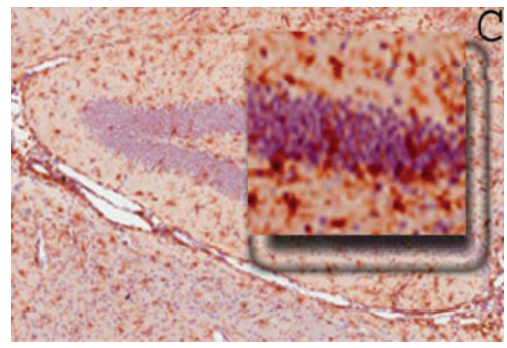

(c)

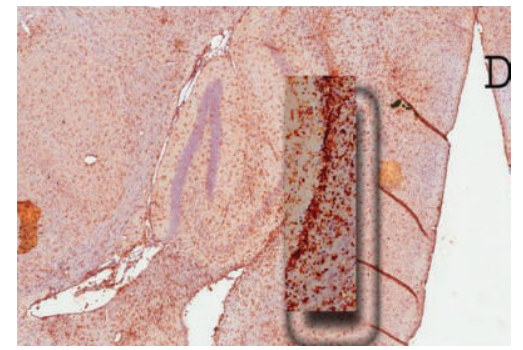

(d)

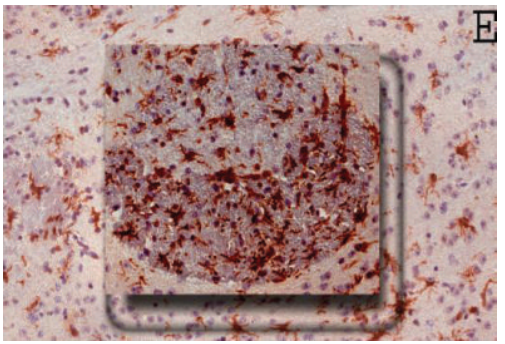

(e)

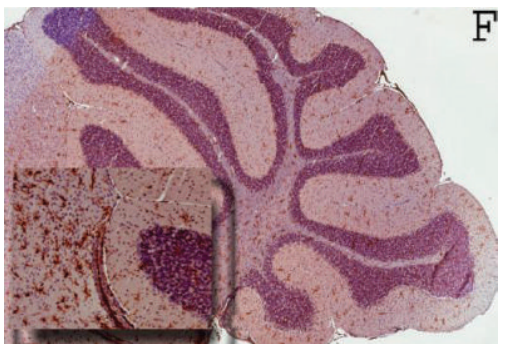

(f)

FIGURE 3: Accumulation of activated amoeboid microglia in the inflamed brain of RSA59 infected Mice. 5-micron thick RSA59 infected Brain sections were immunostained with Ibal and distribution of activated microglia are shown in different region of inflamed brain. Olfactory bulb (a) (200x); basal Fore (b) (200x); hippocampal region (c) (100x); corpus callosum (d) (36x); anterior commisure (e) (200x); deep cerebellar white matter (f) (40x). All images were taken from unpublished data of Das Sarma Laboratory.

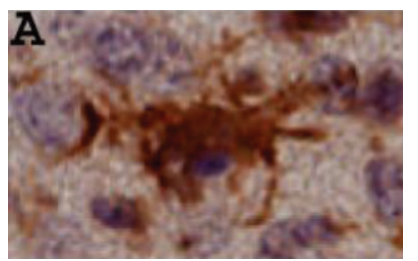

(a)

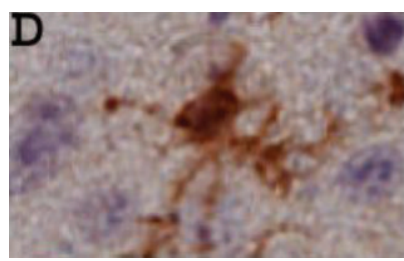

(d)

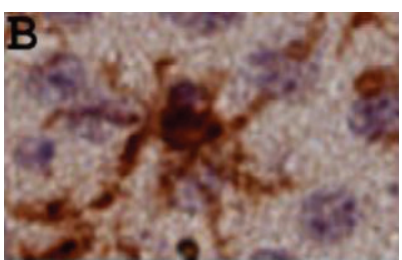

(b)

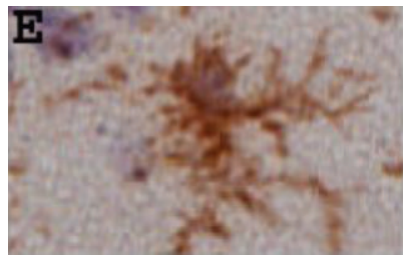

(e)

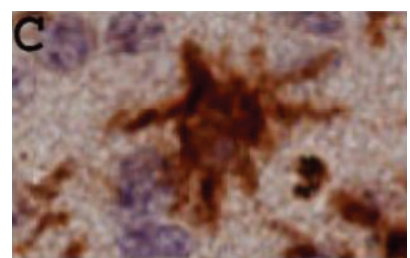

(c)

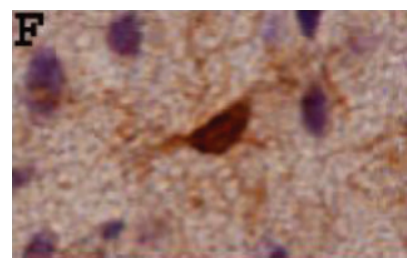

(f)

FIGURE 4: Comparative morphology of activated amoeboid microglia to resting quiescent ramified microglia was taken from acute inflamed brain to resting brain tissue. Different shape of amoeboid microglia, which is embracing neuron immunostained with Ibal and counter stained with haematoxylin (a)-(e) (1000x); (f); quiescent resting Microglia in a nonpathological brain of 4-week-old mice. (Data taken from Das Sarma laboratory; unpublished data). 


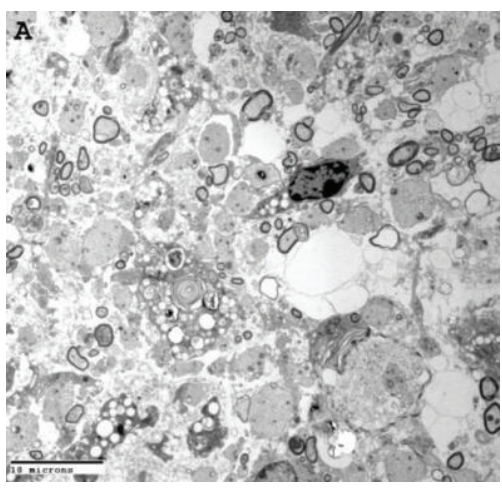

(a)

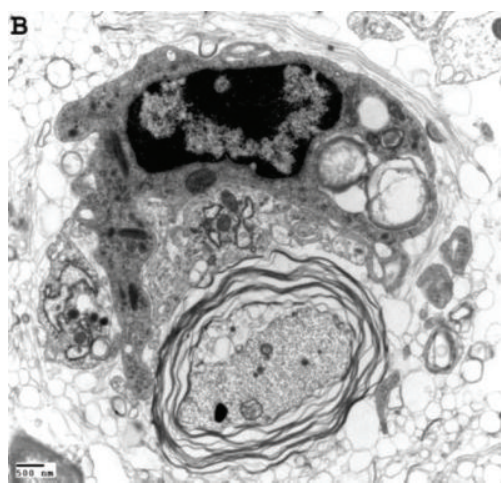

(b)

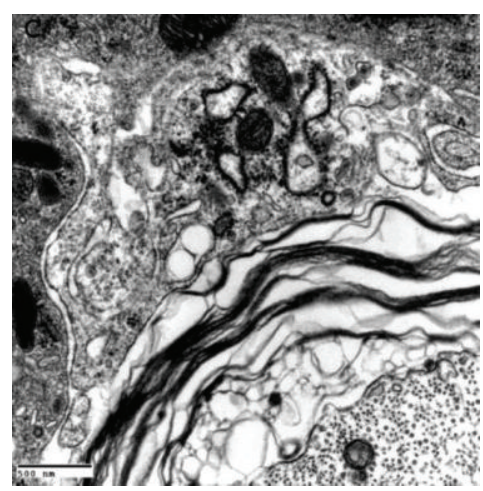

(c)

Figure 5: Ultrastructural appearance of a perineuronal Phagocytic Microglial cell in the chronic demyelinating plaque of RSA59 infected mouse spinal cord. Electron micrograph (a) shows Microglial cells surrounding the demyelinated axon in a demyelinating plaque region of RSA59 infected mouse spinal cord. Microglia has a heterochromatic nucleus with multiple vacuoles. High-magnification images (b) and (c) demonstrates the presence of Microglia surrounding the unraveling myelin sheath in an axon. The Microglia cell membrane is in intimate contact with the outer portion of the myelin sheath as the Microglia is stripping away (phagocytizing) the myelin sheath and engulfs the myelin sheath. Multiple vacuoles with myelin fragments are seen within the cytoplasm of the activated microglia (phagocytotic in nature) - Figure 5 adopted from [12].

All the techniques discussed previously were based on fixed tissues after harvesting. Whereas, in recent period in vivo imaging has made feasible the prospects of monitoring cellular events associated with Neurodegenerative diseases using noninvasive techniques of monitoring [23]. Positron emission tomography (PET) imaging reveals Microglial activation in patients with Neurodegenerative diseases and in animal models by using a specific radio ligand of the peripheral benzodiazepine receptor ([11C]-PK11195), which is upregulated in activated Microglia. Disease-specific localization of Microglial activation can also therefore be detected with PET imaging [24].

Several lines of evidence show that Microglial function is regulated by NADPH oxidase and the production of intracellular reactive oxygen species (ROS). Additionally, the production of Neurotoxic extracellular ROS is also shown to occur primarily through NADPH oxidase, for multiple distinct stimuli, hence, making this enzyme complex could be an ideal therapeutic target. Recently, several peptides, antibiotics, and small molecules have been identified that inhibit NADPH oxidase and are neuroprotective. Combined with early diagnosis through PET-based Microglial imaging, this approach might provide hope for attenuation of the progression of neuroinflammatroy and neurodegenerative disease.

\section{Discussion}

The discipline of Pathology makes a fundamental distinction between acute and chronic inflammation. Acute inflammation comprises the immediate and early response to an injurious agent and is basically a defensive response that paves the way for repair of the damaged site. The concept of chronic inflammation (as opposed to acute inflammation) is more relevant in the context of understanding CNS disease (as opposed to CNS injury), as the very term "disease" implies chronicity. Still the question remains, whether inhibition of Neuroinflammation can be an effective means of therapeutic interventions. To address these questions it is necessary to learn more about how directly Neuroglial cell responses, more specifically towards interaction of neuron with Microglia induced by injury or insult within the CNS and also the mechanism by which these responses ultimately contribute to Neuropathology and consequently Neurobiology of diseases.

The term Neuroinflammation classifies where limited Neuronal insults trigger Glial cell activation without breakdown of the Blood Brain barrier and without concomitant Leukocyte/Blood Monocyte infiltration. Several studies have documented inflammatory components in Alzheimer's Disease (AD) [25], Parkinson's Disease (PD) [23], Amyotrophic Lateral Sclerosis (ALS), Multiple Sclerosis (MS), and a growing number of other nervous system Pathologies [26]. Although inflammation may not typically denote an initiating factor in neurodegenerative disease, there is emerging evidence in Animal models that sustained inflammatory responses involving Microglia and Astrocytes contribute to disease progression [26]. Although inducers of inflammation may be generated in a disease-specific manner, there is evidence for a remarkable convergence in the mechanisms responsible for the sensing, transduction, and amplification of inflammatory processes that result in the production of Neurotoxic mediators. The distinct pathways for production of inducers of inflammation-such as Amyloid- $\beta$, asynuclein, mutant SOD1, and myelin peptide mimetic-are likely determinants of the specific Neuropathological features of AD, PD, ALS, and MS.

Even though this paper discusses the Cytomorphological and Cytochemical identification of Microglial states of activation, future studies warrant the characterization of different activation states of microglia by molecular markers. Mounting lines of evidence of an inflammatory response 
in $\mathrm{AD}$ consist of changes in Microglial morphology-from Ramified (resting) to Amoeboid (active) - and Gliosis to Astrogliosis (manifested by an increase in the number and size of Astrocytes) surrounding the senile plaques. Microglia surrounding Plaques, stain positive for MHC class II, Cox-2, MCP-1, TNF-a, IL-1b, and IL-6 [25]. MCP-1 are the activation markers and proinflammatory mediators of inflammation. Along with that, it is also known to induce the Chemo taxis of Astrocytes and contributes to the recruitment of Astrocytes around senile plaques [27]. Parkinson's disease is now accepted to have an inflammatory component, though originally thought to be a disease characterized with loss of one Neuronal type [28-30]. Reactive Microglia expressing Human Leukocyte Antigen (HLA)-DR and CD11b, along with Lewy bodies, are found in the substantia nigra of PD patients [31]. Prominent neuroinflammation can be readily observed in pathologically affected areas of the CNS and in spinal cords in both Human ALS patients and mouse models of the ALS disease [32].

In addition to the previous markers, elevated levels of chemokine and cytokines and their receptor, like IL-la, CXCR2, CCR3, CCR5, and TGF- $\beta$, have been reported in postmortem of $\mathrm{AD}$ patients brains [33]. Increased levels of colony-stimulating factor (CSF) [30] in the blood have also been reported in PD patients. Activation of Glia in ALS has been extensively characterized and marked with elevated production of potentially cytotoxic molecules such as ROS, inflammatory mediators like COX-2, and pro-inflammatory cytokines like IL-1 $\beta$, TNF- $\alpha$, and IL-6.

Very few parts of literature discussed the role of Glial cells in Neurological diseases or disorders previously. With the understanding of the role of Astrocytes and Microglia in maintaining the homeostasis in the healthy CNS, has been the main attention of the researcher drawn toward Microglia-driven Neuroinflammation. Mounting evidences demonstrate that Chronic Microglial activation is an important component of Neurodegenerative diseases, and this chronic Neuroinflammatory component contributes to Neuronal dysfunction, injury, and loss (and hence to disease progression). The recognition of microglia as the CNS intrinsic immune system, and the understanding of the chronic activation of the system lead to pathologic squeal and lead towards the modern concept of Neuroinflammation. Advancement in staining techniques and in microscopes and techniques of microscopy has extended the older vision of passive Glial responses that were known for a long time as "Reactive Gliosis" to Microglial Neuroinflammation.

Though recent evidence demonstrate the beneficial and neuroprotective profile of Microglia, the acute Microglial activation is deleterious and it is considered that Microglia may also be involved in maintenance, repair, and possibly protection. A major unresolved question is whether inhibition to these responses will be a safe and effective means of reversing or slowing the disease course. Therefore, the ideal therapeutic approach would involve early attenuation of the Microglial responses to levels that are no longer deleterious, rather than the elimination of the Microglial response altogether. Hence, identification of the activation state of Microglia is very important in the light of therapeutic intervention. Hence, Microglial studies emphasizing towards the identification of Microglial states gradually have become significant and therefore many researchers contributed in major ways of identification of Microglia (both resting and activated) using different techniques of Cytomorphological, and Cytochemical staining.

Immunostaining is much more efficient over the other chemical staining method. Most commonly used antibody is $\mathrm{CD} 11 \mathrm{~B}$, which is integrin alpha chain family protein. Integrin alpha-M/beta-2 is implicated in various adhesive interactions of Monocytes, Macrophages, and Granulocytes, as well as in mediating the uptake of complement-coated particles. It is identical with CR-3, the receptor for the iC3b fragment of the third complement component. It probably recognizes the R$\mathrm{G}-\mathrm{D}$ peptide in C3b. Integrin alpha-M/beta-2 is also a receptor for Fibrinogen, factor X, and ICAM1. It recognizes P1 and P2 peptides of Fibrinogen Gamma chain. It is predominantly expressed in monocytes and granulocytes and not specific for tissue Macrophages and more specifically to Microglia. CD11B tends to label endothelial cells in the CNS Blood microvessels which also sometimes make the identification difficult and inaccurate. Whereas, Ibal allograft inflammatory factor-1 family of proteins and are specific to Microlgia and Macrophages, but are not cross reactive with Neurons and Astrocytes in the CNS. It is also known not to cross react with granulocytes or blood microvasculature endothelial cells. Level of Ibal protein expression increases with infection and inflammation within the CNS and with altered level of Calcium ion as it has calcium binding properties. Calcium ions are known to be the one of the important signal residues in all the cells including CNS cells. Calcium ion exerts their signaling activity through association with various calcium binding proteins, many of which are classified into a large protein family, the EF hand protein family (FEBS Latter). Ibal is also an actin-binding protein that enhances membrane ruffling and RAC activation. It also enhances the Actinbundling activity of LCP1 which binds calcium and plays a major role in RAC signaling and in Phagocytosis. It may also play a role in Macrophages activation and function.

In summary, advances made in Cytomorphological and Cytochemical ways of identifying different states of Microglia and other Glial cells have proven to contribute in the field of Neuroimmunology as a whole.

\section{Acknowledgments}

This work was supported by a Research Grant, from Department of Biotechnology (DBT) (BT/PR14260/MED/ 30/437/2010); India, Indian Institute of Science Education and Research-Kolkata (IISER-K), India start up Fund, and Research Grant RG3774A2/1 from the National Multiple Sclerosis Society, to Das Sarma, Koushik Chatterjee and Himadri Dinda are supported by DST funded to RS, Subhajit Das Sarma is supported by DBT funded to JDS and Dhriti Chatterjee is supported by University Grant Commission (UGC), India. The authors thank Central Animal Facility and Dr. S. G. Ramachandra, IISC Bangalore, India for providing experimental mice. The authors thank Dr. Lawrence $\mathrm{C}$ Kenyon, Thomas Jefferson University, USA for his assistance 
in blind studies of pathological samples. The authors have no financial conflict of interest to disclose.

\section{References}

[1] K. D. Barron, "The microglial cell. A historical review," Journal of the Neurological Sciences, vol. 134, no. 1, pp. 57-68, 1995.

[2] M. A. Cuadros and J. Navascués, "The origin and differentiation of microglial cells during development," Progress in Neurobiology, vol. 56, no. 2, pp. 173-189, 1998.

[3] M. A. Cuadros, A. Moujahid, A. Quesada, and J. Navascues, "Development of microglia in the quail optic tectum," Journal of Comparative Neurology, vol. 348, no. 2, pp. 207-224, 1994.

[4] D. M. Reid, V. H. Perry, P.-B. Andersson, and S. Gordon, "Mitosis and apoptosis of microglia in vivo induced by an anti-CR3 antibody which crosses the blood-brain barrier," Neuroscience, vol. 56, no. 3, pp. 529-533, 1993.

[5] R. B. Rock, G. Gekker, S. Hu et al., "Role of microglia in central nervous system infections," Clinical Microbiology Reviews, vol. 17, no. 4, pp. 942-964, 2004.

[6] L. J. Lawson, V. H. Perry, and S. Gordon, "Turnover of resident microglia in the normal adult mouse brain," Neuroscience, vol. 48, no. 2, pp. 405-415, 1992.

[7] Y. Matsumoto, K. Ohmori, and M. Fujiwara, "Immune regulation by brain cells in the central nervous system: microglia but not astrocytes present myelin basic protein to encephalitogenic T cells under in vivo-mimicking conditions," Immunology, vol. 76, no. 2, pp. 209-216, 1992.

[8] E. Lavi, D. H. Gilden, and Z. Wroblewska, "Experimental demyelination produced by the A59 strain of mouse hepatitis virus," Neurology, vol. 34, no. 5, pp. 597-603, 1984.

[9] J. D. Sarma, L. Fu, S. T. Hingley, and E. Lavi, "Mouse hepatitis virus type- 2 infection in mice: an experimental model system of acute meningitis and hepatitis," Experimental and Molecular Pathology, vol. 71, no. 1, pp. 1-12, 2001.

[10] J. Das Sarma, L. Fu, S. T. Hingley, M. M. C. Lai, and E. Lavi, "Sequence analysis of the S gene of recombinant MHV-2/A59 coronaviruses reveals three candidate mutations associated with demyelination and hepatitis," Journal of Neuro Virology, vol. 7, no. 5, pp. 432-436, 2001.

[11] D. Chatterjee, K. Biswas, S. Nag, S. G. Ramachandra, and J. D. Sarma, "Microglia play a major role in direct viral-induced demyelination," Clinical and Developmental Immunology, vol. 2013, Article ID 510396, 12 pages, 2013.

[12] J. Das Sarma, L. C. Kenyon, S. T. Hingley, and K. S. Shindler, "Mechanisms of primary axonal damage in a viral model of multiple sclerosis," Journal of Neuroscience, vol. 29, no. 33, pp. 10272-10280, 2009.

[13] W. Penfield, "A method of staining oligodendroglia and microglia (combined method)," The American Journal of Pathology, vol. 4, no. 2, pp. 153-157, 1928.

[14] D. S. Russell, "Intravital staining of microglia with trypan blue," The American Journal of Pathology, vol. 5, no. 5, pp. 451-458, 1929.

[15] M. Gencic and M. Oehmichen, "A modification of microglia impregnation," Microscopica Acta, vol. 82, no. 3, pp. 201-206, 1979.
[16] T. Scott, "A silver impregnation method for reactive microglia in $1 \mu \mathrm{m}$ epoxy sections," Acta Neuropathologica, vol. 46, no. 1-2, pp. $155-158,1979$.

[17] H. Kettenmann, U. K. Hanisch, M. Noda, and A. Verkhratsky, "Physiology of microglia," Physiological Reviews, vol. 91, no. 2, pp. 461-553, 2011.

[18] M. Oehmichen, H. Wiethoelter, and M. Gencic, "Cytochemical markers for mononuclear phagocytes as demonstrated in reactive microglia and globoid cells," Acta Histochemica, vol. 66, no. 2, pp. 243-252, 1980.

[19] K. S. Shindler, L. C. Kenyon, M. Dutt, S. T. Hingley, and J. Das Sarma, "Experimental optic neuritis induced by a demyelinating strain of mouse hepatitis virus," Journal of Virology, vol. 82, no. 17, pp. 8882-8886, 2008.

[20] K. S. Shindler, D. Chatterjee, K. Biswas et al., "Macrophage-mediated optic neuritis induced by retrograde axonal transport of spike gene recombinant mouse hepatitis virus," Journal of Neuropathology and Experimental Neurology, vol. 70, no. 6, pp. 470480, 2011.

[21] H. Kanazawa, K. Ohsawa, Y. Sasaki, S. Kohsaka, and Y. Imai, "Macrophage/microglia-specific protein Ibal enhances membrane ruffling and Rac activation via phospholipase C- $\gamma$ dependent pathway," Journal of Biological Chemistry, vol. 277, no. 22, pp. 20026-20032, 2002.

[22] G. W. Simmons, W. W. Pong, R. J. Emnett et al., "Neurofibromatosis-1 heterozygosity increases microglia in a spatially and temporally restricted pattern relevant to mouse optic glioma formation and growth," Journal of Neuropathology and Experimental Neurology, vol. 70, no. 1, pp. 51-62, 2011.

[23] P. Damier, E. C. Hirsch, P. Zhang, Y. Agid, and F. Javoy-Agid, "Glutathione peroxidase, glial cells and Parkinson's disease," Neuroscience, vol. 52, no. 1, pp. 1-6, 1993.

[24] A. Cagnin, D. J. Brooks, A. M. Kennedy et al., "In-vivo measurement of activated microglia in dementia," The Lancet, vol. 358, no. 9280, pp. 461-467, 2001.

[25] H. Akiyama, S. Barger, S. Barnum et al., "Inflammation and Alzheimer's disease," Neurobiology of Aging, vol. 21, no. 3, pp. 383$421,2000$.

[26] C. K. Glass, K. Saijo, B. Winner, M. C. Marchetto, and F. H. Gage, "Mechanisms Underlying Inflammation in Neurodegeneration," Cell, vol. 140, no. 6, pp. 918-934, 2010.

[27] T. Wyss-Coray, J. D. Loike, T. C. Brionne et al., "Adult mouse astrocytes degrade amyloid- $\beta$ in vitro and in situ," Nature Medicine, vol. 9, no. 4, pp. 453-457, 2003.

[28] M. L. Block and J. S. Hong, "Chronic microglial activation and progressive dopaminergic neurotoxicity," Biochemical Society Transactions, vol. 35, no. 5, pp. 1127-1132, 2007.

[29] P. L. McGeer and E. G. McGeer, "Glial reactions in Parkinson's disease," Movement Disorders, vol. 23, no. 4, pp. 474-483, 2008.

[30] T. Nagatsu and M. Sawada, "Inflammatory process in Parkinson's disease: role for cytokines," Current Pharmaceutical Design, vol. 11, no. 8, pp. 999-1016, 2005.

[31] P. L. McGeer, S. Itagaki, B. E. Boyes, and E. G. McGeer, "Reactive microglia are positive for HLA-DR in the substantia nigra of Parkinson's and Alzheimer's disease brains," Neurology, vol. 38, no. 8, pp. 1285-1291, 1988.

[32] P. L. McGeer and E. G. McGeer, "Inflammatory processes in amyotrophic lateral sclerosis," Muscle and Nerve, vol. 26, no. 4, pp. 459-470, 2002. 
[33] L. Cartier, O. Hartley, M. Dubois-Dauphin, and K.-H. Krause, "Chemokine receptors in the central nervous system: role in brain inflammation and neurodegenerative diseases," Brain Research Reviews, vol. 48, no. 1, pp. 16-42, 2005. 


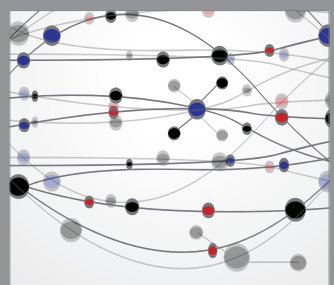

The Scientific World Journal
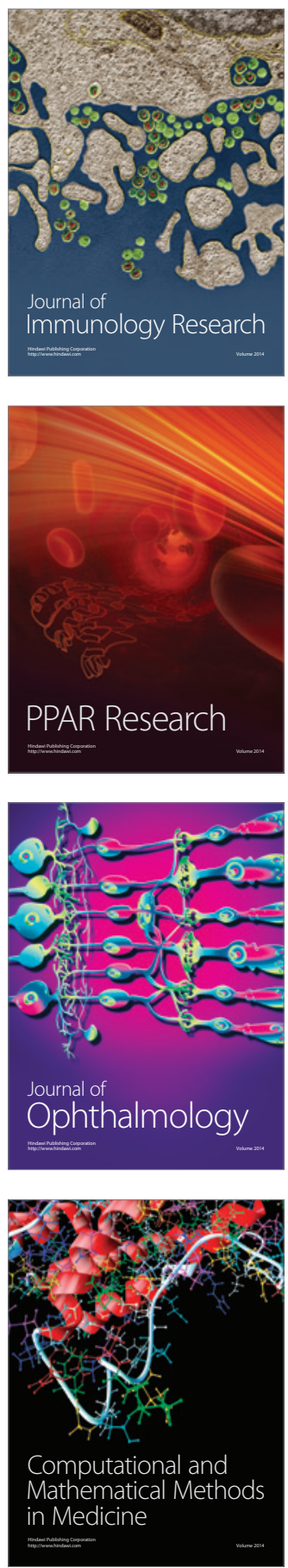

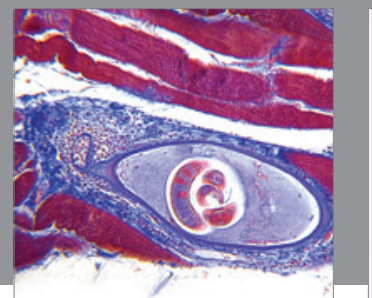

Gastroenterology

Research and Practice
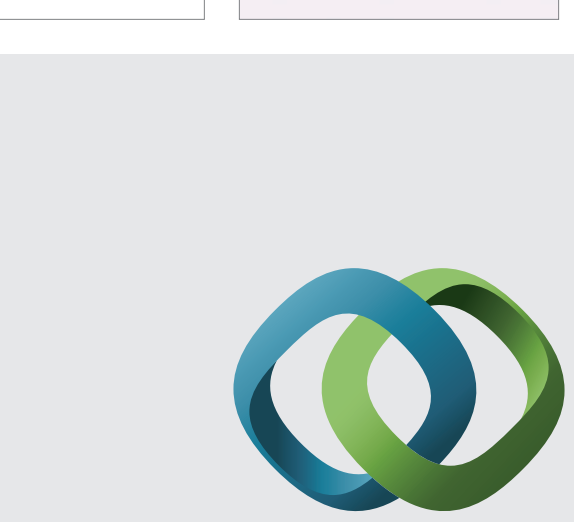

\section{Hindawi}

Submit your manuscripts at

http://www.hindawi.com
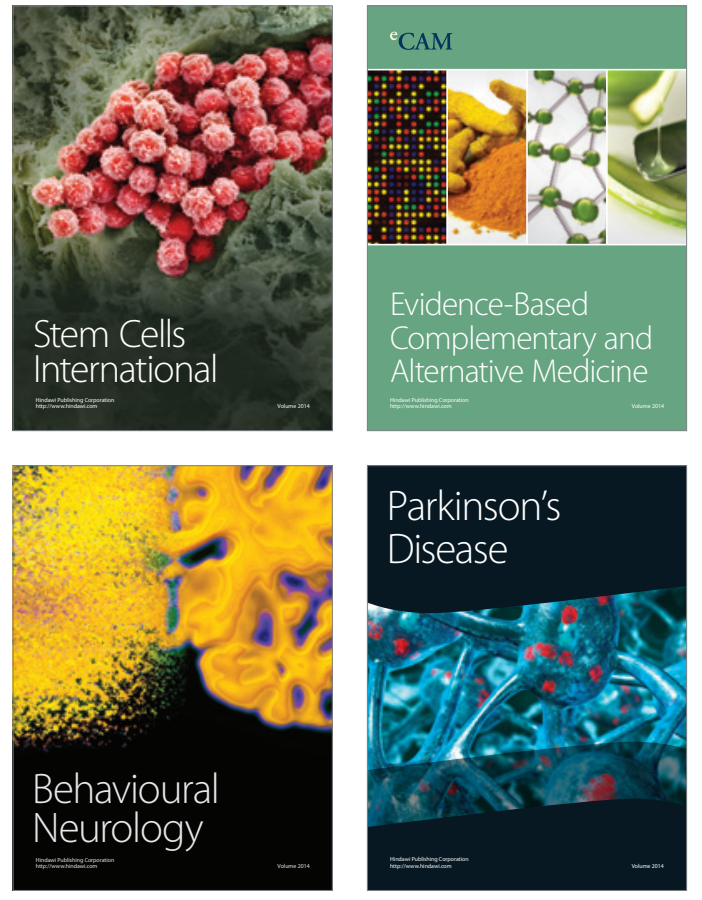
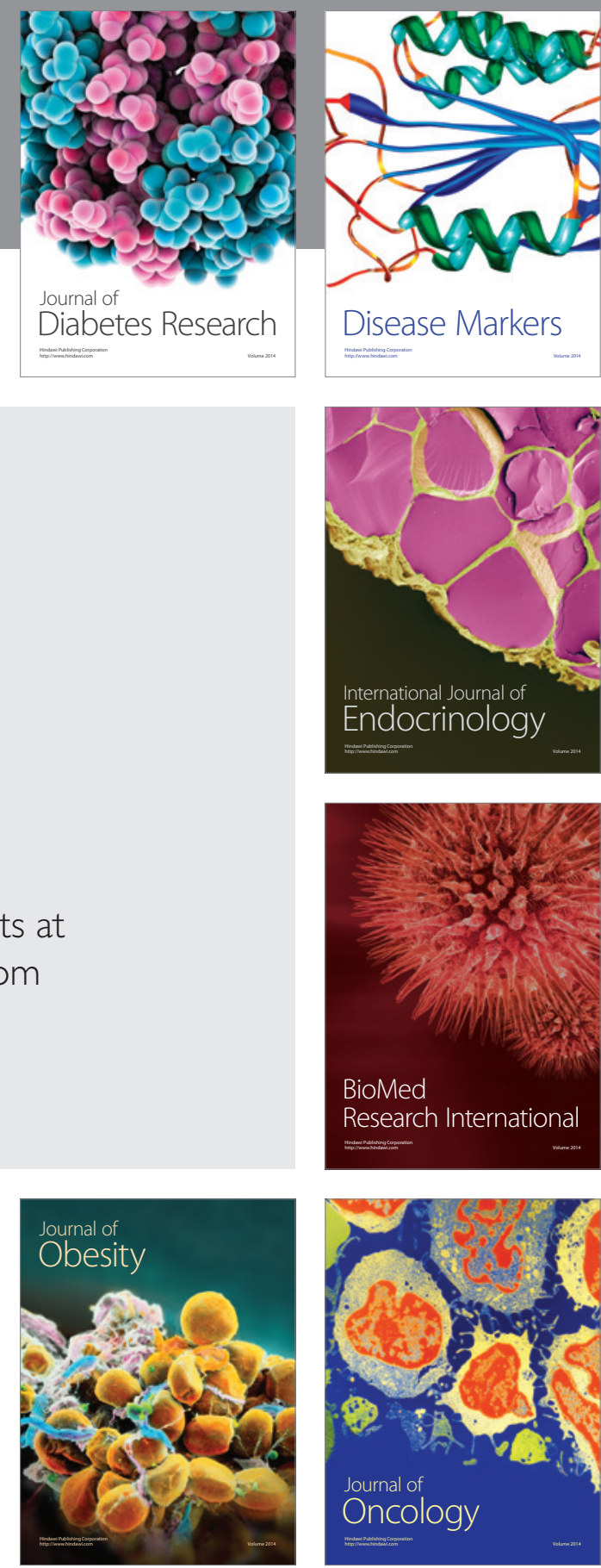

Disease Markers
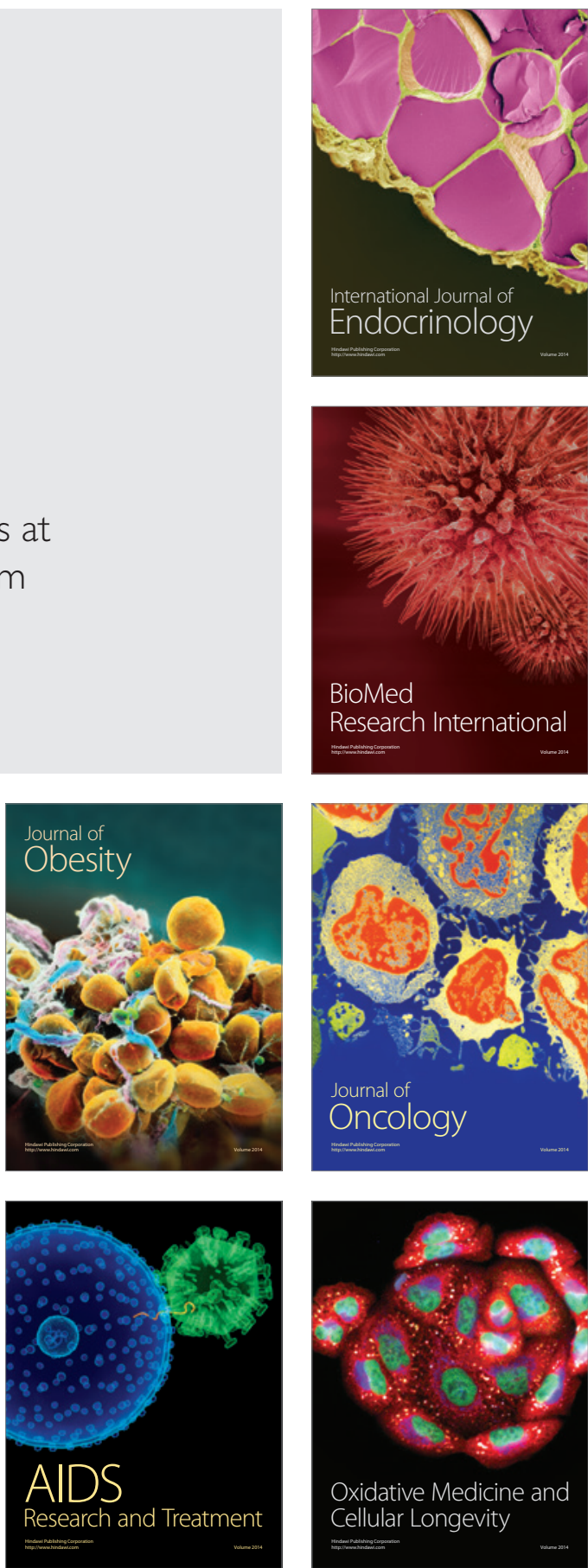